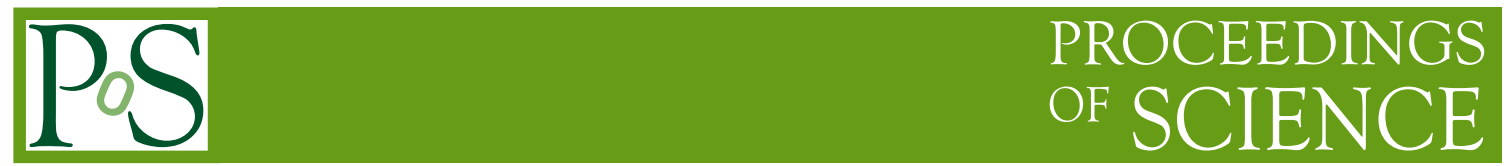

\title{
Studies of excited heavy flavor states at CMS
}

\author{
Nikita Petrov on behalf of the CMS Collaboration ${ }^{a, *}$ \\ ${ }^{a}$ Moscow Institute of Physics and Technology (MIPT), \\ Dolgoprudny, Russia \\ E-mail: nikita.petrov@cern.ch
}

The measurement of $\mathrm{B}_{\mathrm{c}}^{+}(2 \mathrm{~S})$ and $\mathrm{B}_{\mathrm{c}}^{+*}(2 \mathrm{~S})$ cross section ratios by the CMS experiment is reported, as well as the observation of a new excited beauty strange baryon decaying to $\Xi_{\mathrm{b}}^{-} \pi^{+} \pi^{-}$final state. Both analyses use proton-proton collision data collected at $\sqrt{s}=13 \mathrm{TeV}$.

*** The European Physical Society Conference on High Energy Physics (EPS-HEP2021), *** *** 26-30 July $2021 * * *$

*** Online conference, jointly organized by Universität Hamburg and the research center DESY ***

\footnotetext{
${ }^{*}$ Speaker
} 


\section{Introduction}

Studies of the excited heavy flavour states are extremely important for testing mechanisms responsible for the dynamics of quarks and hadron formation. Spectroscopy and cross section measurements of the states containing heavy-flavour quarks can probe various theoretical predictions. Different models predict a spectrum of excited $\Xi_{\mathrm{b}}$ baryons $[1,2]$, as well as a family of $\mathrm{B}_{\mathrm{c}}^{+}$ mesons [3] (charge-conjugate states are also implied in the whole paper). Large production of $b \bar{b}$ pairs at the CERN LHC allows the CMS, LHCb and ATLAS experiments to perform the studies of the excited states even if its production cross section is small.

The present work reports recent results in excited heavy flavour states physics at CMS [4]: the measurement of $\mathrm{B}_{\mathrm{c}}^{+}(2 \mathrm{~S})$ and $\mathrm{B}_{\mathrm{c}}^{+*}(2 \mathrm{~S})$ cross section ratios [5] and the observation of a new excited baryon $\Xi_{\mathrm{b}}(6100)^{-}$decaying into $\Xi_{\mathrm{b}}^{-} \pi^{+} \pi^{-}$final state [6]. Both analyses were performed using proton-proton collision data collected at $\sqrt{s}=13 \mathrm{TeV}$ by the CMS experiment at the LHC, corresponding to an integrated luminosity of $143 \mathrm{fb}^{-1}$ for [5] and $140 \mathrm{fb}^{-1}$ for [6].

\section{Measurement of $B_{c}^{+}(2 S)$ and $B_{c}^{+*}(2 S)$ cross section ratios}

$\mathrm{B}_{\mathrm{c}}^{+}$is a charged meson consisting of two heavy quarks, beauty and charm ones. The spectrum of this state is predicted to be very populated, but spectroscopic observations are poor so far. The production yields of this state are significantly smaller than for charmonium and bottomonium states, due to proportionality of production cross section to $\alpha_{S}^{4}$. Nevertheless, the $\mathrm{B}_{\mathrm{c}}^{+}$family is placed between the charmonium and bottomonium systems, therefore many properties can be predicted theoretically. However, inequality of masses and velocities of charm and beauty quarks can lead to more complex dynamics inside the states. Detailed and careful studies of $\mathrm{B}_{\mathrm{c}}^{+}$system allow to probe theoretical models and improve our knowledge of its nature.

In 2014 the ATLAS Collaboration observed a new state with mass consistent to predictions for $\mathrm{B}_{\mathrm{c}}^{+}(2 \mathrm{~S})$ state in $\mathrm{B}_{\mathrm{c}}^{+} \pi^{+} \pi^{-}$system [7]. However, LHCb, using $8 \mathrm{TeV}$ data, did not find any significant signal in the same mass region [8]. Then, using full RunII data, the CMS Collaboration observed two well resolved peaks [9], $\mathrm{B}_{\mathrm{c}}^{+}(2 \mathrm{~S})$ and $\mathrm{B}_{\mathrm{c}}^{+*}(2 \mathrm{~S})$. Both states are reconstructed in the $\mathrm{B}_{\mathrm{c}}^{+} \pi^{+} \pi^{-}$final state, where $\mathrm{B}_{\mathrm{c}}^{+}(2 \mathrm{~S})$ decays directly into $\mathrm{B}_{\mathrm{c}}^{+} \pi^{+} \pi^{-}, \mathrm{B}_{\mathrm{c}}^{+*}(2 \mathrm{~S})$ first goes to $\mathrm{B}_{\mathrm{c}}^{+*} \pi^{+} \pi^{-}$and then $\mathrm{B}_{\mathrm{c}}^{+*}$ decays to the ground state emitting a soft, not identified, photon. Since theory predicts $\Delta \mathrm{M}=\left[\mathrm{M}\left(\mathrm{B}_{\mathrm{c}}^{+*}\right)-\mathrm{M}\left(\mathrm{B}_{\mathrm{c}}^{+}\right)\right]-\left[\mathrm{M}\left(\mathrm{B}_{\mathrm{c}}^{+*}(2 \mathrm{~S})\right)-\mathrm{M}\left(\mathrm{B}_{\mathrm{c}}^{+}(2 \mathrm{~S})\right)\right]=20 \mathrm{MeV}, \mathrm{B}_{\mathrm{c}}^{+*}(2 \mathrm{~S})$ is placed below the $\mathrm{B}_{\mathrm{c}}^{+}(2 \mathrm{~S})$ state. Two variables were measured in this study: $\mathrm{M}\left(\mathrm{B}_{\mathrm{c}}^{+}(2 \mathrm{~S})\right)=6871.0 \pm 1.2 \pm 0.8 \pm 0.8$ $\mathrm{MeV}$ and $\Delta \mathrm{M}=29.1 \pm 1.5 \pm 0.7 \mathrm{MeV}$. Recently, the LHCb Collaboration confirmed two-peaks structure using RunI and RunII statistics [10], the results are in a good agreement with CMS. The next logical step is to measure cross section ratios of the observed states.

The $\mathrm{B}_{\mathrm{c}}^{+}$ground state is reconstructed in the $\mathrm{J} / \psi \pi^{+}$mode through a kinematic vertex fit, combining the dimuon with another track with pion mass hypothesis. The dimuon invariant mass is constrained to the world-average $\mathrm{J} / \psi$ mass [11]. For the cross section ratio the $\mathrm{B}_{\mathrm{c}}^{+}$candidates are required to belong to the kinematic region $\mathrm{p}_{\mathrm{T}}>15 \mathrm{GeV}$ and $|y|<2.4$.

To reconstruct excited $\mathrm{B}_{\mathrm{c}}^{+}$candidates, ground state is combined with two opposite-sign (OS) tracks originating from the primary vertex $(\mathrm{PV})$. To improve mass resolution the following mass variable is used in this analysis $\mathrm{M}\left(\mathrm{B}_{\mathrm{c}}^{+} \pi^{+} \pi^{-}\right)-\mathrm{M}\left(\mathrm{B}_{\mathrm{c}}^{+}\right)+\mathrm{M}_{\mathrm{B}_{\mathrm{c}}^{+}}^{\mathrm{PDG}}$. 
The $\mathrm{J} / \psi \pi^{+}$invariant mass distributions is presented in Fig. 1(left). The spectrum is described by several components: combinatorial backround is parametrized by a first-order polynomial, the contribution from the partially reconstructed $\mathrm{B}_{\mathrm{c}}^{+} \rightarrow \mathrm{J} / \psi \pi^{+} X$ decays is modeled by an ARGUS function convolved with Gaussian resolution and the signal is described by a double Gaussian function. In addition the spectrum contains a small contribution from $\mathrm{B}_{\mathrm{c}}^{+} \rightarrow \mathrm{J} / \psi \mathrm{K}^{+}$decays, for which the shape is determined from Monte-Carlo (MC) simulation studies and the normalization is scaled by the ratio of the corresponding branching fractions and the reconstruction efficiencies. From an unbinned extended maximum-likelihood (UEML) fit the $\mathrm{B}_{\mathrm{c}}^{+}$yield is extracted to be $7629 \pm 225$ (stat.).
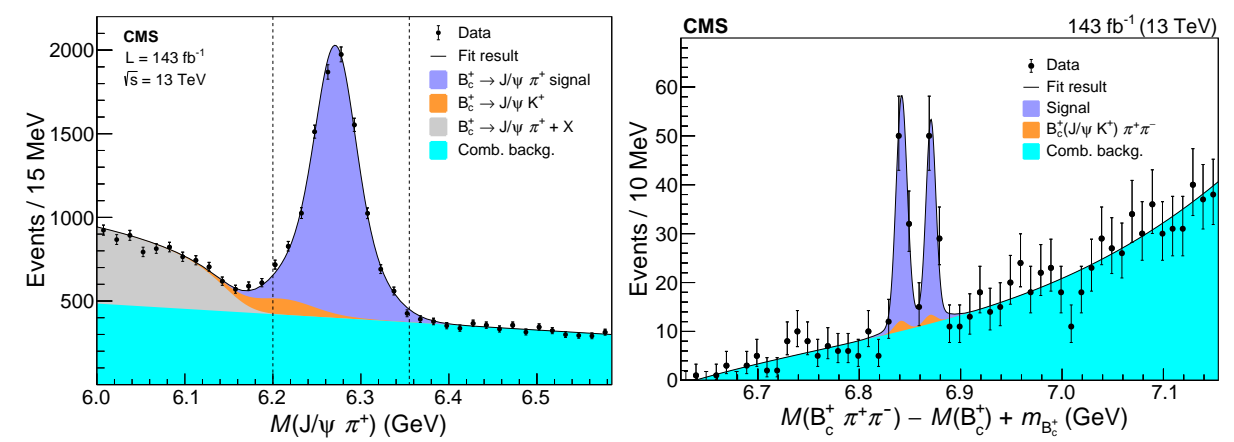

Figure 1: Invariant mass distribution of $\mathrm{J} / \psi \pi^{+}$(left) and $\mathrm{B}_{\mathrm{c}}^{+} \pi^{+} \pi^{-}$(right) with fits, details are listed in the text [5].

The $\mathrm{B}_{\mathrm{c}}^{+} \pi^{+} \pi^{-}$spectrum is shown in Fig. 1(right), both signal components are described by double Gaussian functions, the combinatorial background is parametrized by third-order Chebyshev polynomial and the background contributions arising from the $\mathrm{B}_{\mathrm{c}}^{+} \rightarrow \mathrm{J} / \psi \mathrm{K}^{+}$decays are considered with shapes identical to signal. The UEML fit gives $67 \pm 10$ and $52 \pm 9$ candidates for the lowerand higher-mass peaks, respectively.

The ratios of the $\mathrm{B}_{\mathrm{c}}^{(*)}(2 S)^{+}$to $\mathrm{B}_{\mathrm{c}}^{+}$and $\mathrm{B}_{\mathrm{c}}^{+*}(2 \mathrm{~S})$ to $\mathrm{B}_{\mathrm{c}}^{+}(2 \mathrm{~S})$ cross sections, $R^{*+}, R^{+}$and $R^{*+} / R^{+}$, respectively are obtained from the ratios of the measured yields $N$, corrected by the detection efficiencies $\epsilon$, using the following equations:

$$
\begin{aligned}
& R^{+}=\frac{\sigma\left(\mathrm{B}_{\mathrm{c}}^{+}(2 \mathrm{~S})\right)}{\sigma\left(\mathrm{B}_{\mathrm{c}}^{+}\right)} \mathcal{B}\left(\mathrm{B}_{\mathrm{c}}^{+}(2 \mathrm{~S}) \rightarrow \mathrm{B}_{\mathrm{c}}^{+} \pi^{+} \pi^{-}\right)=\frac{N\left(\mathrm{~B}_{\mathrm{c}}^{+}(2 \mathrm{~S})\right)}{N\left(\mathrm{~B}_{\mathrm{c}}^{+}\right)} \frac{\epsilon\left(\mathrm{B}_{\mathrm{c}}^{+}\right)}{\epsilon\left(\mathrm{B}_{\mathrm{c}}^{+}(2 \mathrm{~S})\right)}=(3.47 \pm 0.63 \pm 0.33) \% \\
& R^{+*}=\frac{\sigma\left(\mathrm{B}_{\mathrm{c}}^{+*}(2 \mathrm{~S})\right)}{\sigma\left(\mathrm{B}_{\mathrm{c}}^{+}\right)} \mathcal{B}\left(\mathrm{B}_{\mathrm{c}}^{+*}(2 \mathrm{~S}) \rightarrow \mathrm{B}_{\mathrm{c}}^{+*} \pi^{+} \pi^{-}\right)=\frac{N\left(\mathrm{~B}_{\mathrm{c}}^{+*}(2 \mathrm{~S})\right)}{N\left(\mathrm{~B}_{\mathrm{c}}^{+}\right)} \frac{\epsilon\left(\mathrm{B}_{\mathrm{c}}^{+}\right)}{\epsilon\left(\mathrm{B}_{\mathrm{c}}^{+*}(2 \mathrm{~S})\right)}=(4.69 \pm 0.71 \pm 0.56) \% \\
& R^{*+} / R^{+}=\frac{\sigma\left(\mathrm{B}_{\mathrm{c}}^{+*}(2 \mathrm{~S})\right)}{\sigma\left(\mathrm{B}_{\mathrm{c}}^{+}(2 \mathrm{~S})\right)} \frac{\mathcal{B}\left(\mathrm{B}_{\mathrm{c}}^{+*}(2 \mathrm{~S}) \rightarrow \mathrm{B}_{\mathrm{c}}^{+*} \pi^{+} \pi^{-}\right)}{\mathcal{B}\left(\mathrm{B}_{\mathrm{c}}^{+}(2 \mathrm{~S}) \rightarrow \mathrm{B}_{\mathrm{c}}^{+} \pi^{+} \pi^{-}\right)}=\frac{N\left(\mathrm{~B}_{\mathrm{c}}^{+*}(2 \mathrm{~S})\right)}{N\left(\mathrm{~B}_{\mathrm{c}}^{+}(2 \mathrm{~S})\right)} \frac{\epsilon\left(\mathrm{B}_{\mathrm{c}}^{+}(2 \mathrm{~S})\right)}{\epsilon\left(\mathrm{B}_{\mathrm{c}}^{+*}(2 \mathrm{~S})\right)}=1.35 \pm 0.22 \pm 0.33,
\end{aligned}
$$

where the $\mathcal{B}$ parameters are the unknown branching fractions of the $\mathrm{B}_{\mathrm{c}}^{(*)}(2 \mathrm{~S})^{+} \rightarrow \mathrm{B}_{\mathrm{c}}^{(*)+} \pi^{+} \pi^{-}$ decays. The main contributions to the systematic uncertainties are related to the $\mathrm{B}_{\mathrm{c}}^{+} \pi^{+} \pi^{-}$and $\mathrm{J} / \psi \pi^{+}$ fit models, the pion tracking for $R^{+}$and $R^{+*}$ ratios and the $\mathrm{B}_{\mathrm{c}}^{(*)}(2 \mathrm{~S})^{+}$decay kinematics (modelling of dipion mass distributions).

The dependence of the cross section ratios on the kinematics of the $\mathrm{B}_{\mathrm{c}}^{+}$meson were obtained and none of them shows significant variations with the $\mathrm{p}_{\mathrm{T}}$ or $|y|$ in the region of the study. The 
plots are presented in Fig. 2. In addition to the cross section ratios measurement a normalized dipion invariant mass distributions for the $\mathrm{B}_{\mathrm{c}}^{(*)}(2 S)^{+} \rightarrow \mathrm{B}_{\mathrm{c}}^{+} \pi^{+} \pi^{-}$decays were compared with phase space from the Monte-Carlo simulations and some discrepancy between the data and simulation was noticed, however more data is needed for the further studies.
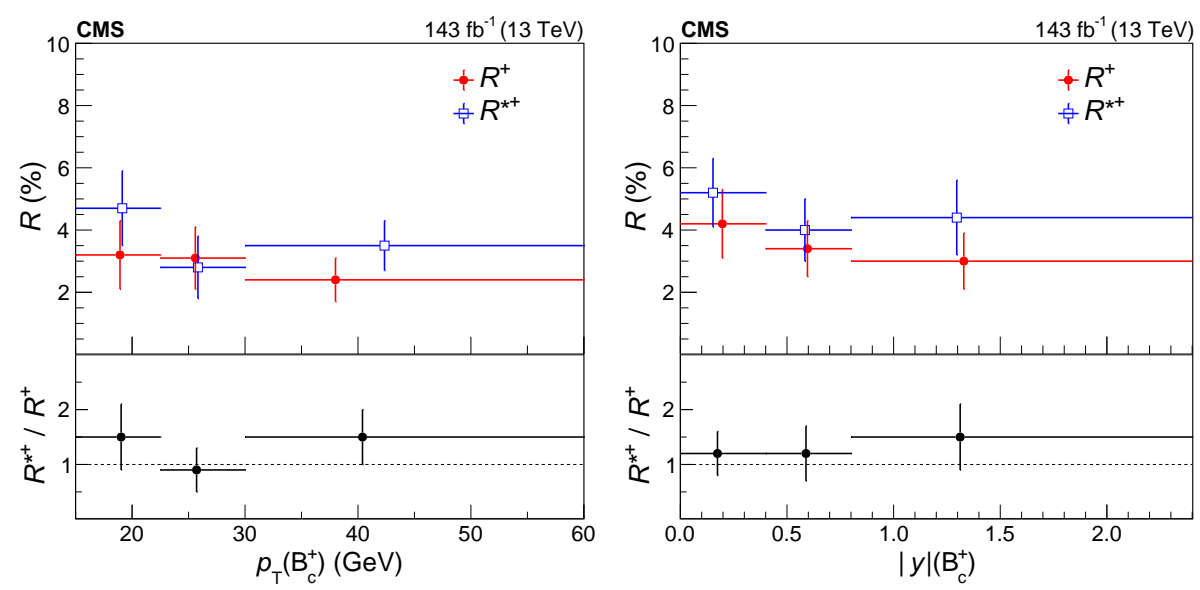

Figure 2: $R^{+}, R^{+*}$ and $R^{*+} / R^{+}$cross section ratios as functions of $\mathrm{B}_{\mathrm{c}}^{+} \mathrm{p}_{\mathrm{T}}$ and $|y|$ [5].

\section{First observation of $\Xi_{b}(6100)^{-}$baryon}

The $\Xi_{\mathrm{b}}^{-}$baryon family consists of isodublet states composed of $b s q$ quarks, where $q$ represents an up or a down quark for the $\Xi_{\mathrm{b}}^{0}$ and $\Xi_{\mathrm{b}}^{-}$states, respectively. Various theoretical models and calculations predict a spectrum of excited $\Xi_{\mathrm{b}}^{-}$baryons. In particular there are several predictions for orbitally excited P-wave $\Xi_{\mathrm{b}}^{* *}$ states with quantum numbers $J^{P}=1 / 2^{-}\left(3 / 2^{-}\right)$, expected to decay to $\Xi_{\mathrm{b}}^{\prime}\left(\Xi_{\mathrm{b}}^{*}\right) \pi$.

Excited states with light diquark angular momentum equal to one have been observed at the CERN LHC in the $\Xi_{\mathrm{b}}^{-} \pi^{+}$and $\Xi_{\mathrm{b}}^{0} \pi^{-}$invariant mass spectra. Recently, the LHCb Collaboration reported the observation of the $\Xi_{\mathrm{b}}(6227)^{-}$and $\Xi_{\mathrm{b}}(6227)^{0}$ states, the former decaying to both $\Lambda_{\mathrm{b}}^{0} \mathrm{~K}^{-}$ and $\Xi_{\mathrm{b}}^{0} \pi^{-}$, and the latter to $\Xi_{\mathrm{b}}^{-} \pi^{+}$.

The present work reports the results of the search for a new $\Xi_{\mathrm{b}}^{* *-} \rightarrow \Xi_{\mathrm{b}}^{* 0} \pi^{-} \rightarrow \Xi_{\mathrm{b}}^{-} \pi^{+} \pi^{-}$ resonance. The ground states are reconstructed via $\mathrm{J} / \psi \Xi^{-}$and $\mathrm{J} / \psi \Lambda \mathrm{K}^{-}$decays, where $\mathrm{J} / \psi \rightarrow \mu^{-} \mu^{+}$, $\Xi^{-} \rightarrow \Lambda \pi^{-}$and $\Lambda \rightarrow \mathrm{p} \pi^{-}$. The reflection $\Xi_{\mathrm{b}}^{-} \rightarrow \mathrm{J} / \psi \Sigma^{0} \mathrm{~K}^{-}$(a photon from $\Sigma^{0} \rightarrow \Lambda \gamma$ is not reconstructed) is also used for the new resonance search. Fig. 3 presents $J / \psi \Xi^{-}$(left) and $J / \psi \Lambda \mathrm{K}^{-}$ invariant mass distributions and UEML fits. Both signal components are described by a double Gaussian function, the background is parametrized by a first-order polynomial for $\mathrm{J} / \psi \Xi^{-}$and an exponential for the $\mathrm{J} / \psi \Lambda \mathrm{K}^{-}$sample. The reflection contribution is taken into account by an asymmetric Gaussian with shape fully fixed from simulation studies. The fits result in a number of signal $\Xi_{\mathrm{b}}^{-}$candidates $859 \pm 36$ and $815 \pm 74$ for $\mathrm{J} / \psi \Xi^{-}$and $\mathrm{J} / \psi \Lambda \mathrm{K}^{-}$channels, respectively; the reflection yield is $820 \pm 158$, where all uncertainties are statistical only.

Then the ground states are combined with opposite-charged pair of pions originating from the PV. To improve the resolution of the $\Xi_{\mathrm{b}}^{-} \pi^{+} \pi^{-}$invariant mass spectrum, all the tracks forming the chosen PV and the $\Xi_{\mathrm{b}}^{-}$candidate are refitted together in a common vertex. The mass difference 

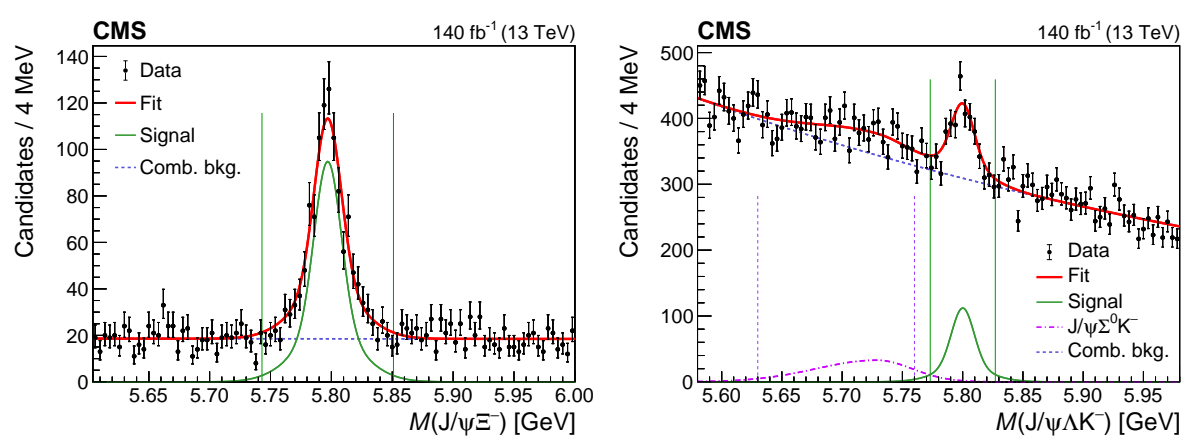

Figure 3: Invariant mass distributions of the selected $\Xi_{\mathrm{b}}^{-}$candidates in the $\mathrm{J} / \psi \Xi^{-}$(left) and $\mathrm{J} / \psi \Lambda \mathrm{K}^{-}$(right) decay channels with the fit results superimposed [6].

variable $\Delta \mathrm{M}=\mathrm{M}\left(\Xi_{\mathrm{b}}^{-} \pi^{+} \pi^{-}\right)-\mathrm{M}\left(\Xi_{\mathrm{b}}^{-}\right)-2 \mathrm{M}_{\pi}^{\mathrm{PDG}}$ is used in this analysis. An additional mass window on $\Xi_{\mathrm{b}}^{-} \pi^{+}$mass is applied to take into account the intermediate $\Xi_{\mathrm{b}}^{* 0}$ resonance.

Fig. 4 shows the $\Delta \mathrm{M}$ distributions for fully (left) and partially (right) reconstructed $\Xi_{\mathrm{b}}^{-}$ground states. Both plots show a narrow peak near the mass threshold. A simultaneous UEML fit is performed on the two data samples. The signal contribution is described by a relativistic BreightWigner function convolved with the resolution, the background component is modeled by the threshold function. The statistical local significance of the $\Xi_{\mathrm{b}}(6100)^{-}$state exceeds 6.2 standard deviations including systematic variations. The measured mass difference of the observed baryon is $\Delta \mathrm{M}=24.14 \pm 0.22$ (stat.) \pm 0.09 (syst.) $\mathrm{MeV}$. Using known mass of the $\Xi_{\mathrm{b}}^{-}$state $\mathrm{M}\left(\Xi_{\mathrm{b}}(6100)^{-}\right)$ is obtained to be $6100.3 \pm 0.2$ (stat.) \pm 0.1 (syst.) $\pm 0.6\left(\Xi_{\mathrm{b}}^{-}\right) \mathrm{MeV}$. With the current statistical power, natural width of the state is consistent with zero, thus upper limit of $1.9 \mathrm{MeV}$ has been established at $95 \%$ confidence level. The systematic uncertainties include variations of the fit range, fit models and slight differences between the data and simulations.
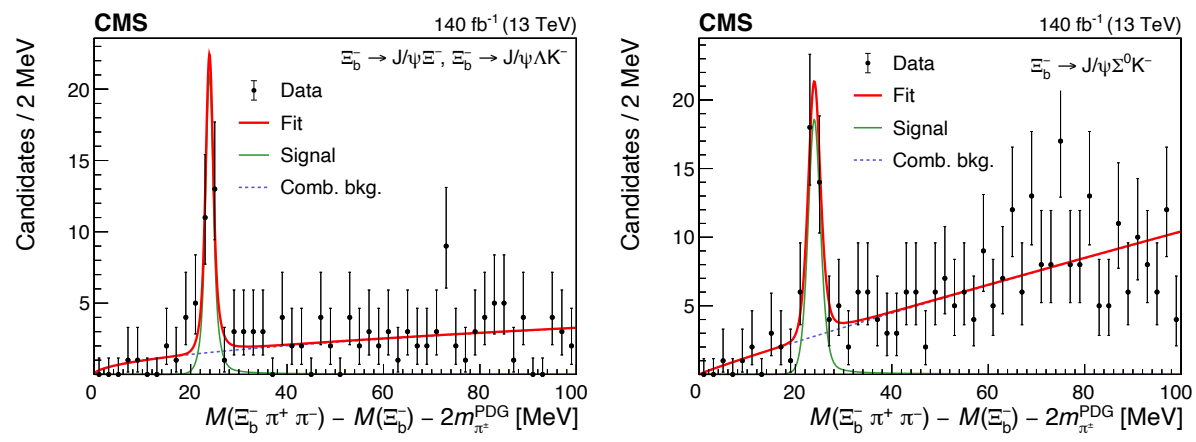

Figure 4: Distributions of the invariant mass difference $\Delta \mathrm{M}$ with fully (left) and partially (right) reconstructed $\Xi_{\mathrm{b}}^{-}$candidates. The result of the simultaneous fit is also presented [6].

\section{Conclusion}

The CMS Collaboration contributes significantly in the study of excited heavy flavor states. Two excited $\mathrm{B}_{\mathrm{c}}^{+}(2 \mathrm{~S})$ and $\mathrm{B}_{\mathrm{c}}^{+*}(2 \mathrm{~S})$ states were at first observed at CMS, and their cross section ratios were measured. In addition, dependencies of the ratios on $\mathrm{B}_{\mathrm{c}}^{+}$kinematics were investigated, as 
well as the dipion invariant mass distributions. Moreover, the new excited $\Xi_{\mathrm{b}}(6100)^{-}$baryon was discovered in $\Xi_{\mathrm{b}}^{-} \pi^{+} \pi^{-}$final state.

\section{Acknowledgement}

The author would like to thank the EPS-HEP 2021 organizers for the interesting program and opportunity to present these important results.

\section{References}

[1] Bing Chen, Ke-Wei Wei, Xiang Liu, and Ailin Zhang, Role of newly discovered $\Xi_{\mathrm{b}}(6227)^{-}$ for constructing excited bottom baryon family, Phys. Rev. D98 (2018 031502)

[2] Yohei Kawakami and Masayasu Harada, Singly heavy baryons with chiral partner structure in a three-flavor chiral model, Phys. Rev. D 99 (2019) 094016

[3] Stephen Godfrey, Spectroscopy of $\mathrm{B}_{c}$ mesons in the relativized quark model, Phys. Rev. D 70 (2004) 054017

[4] CMS Collaboration, The CMS experiment at the CERN LHC, JINST 3 (2008) S08004

[5] CMS Collaboration, Measurement of $\mathrm{B}_{\mathrm{c}}^{+}(2 \mathrm{~S})$ and $\mathrm{B}_{\mathrm{c}}^{+*}(2 \mathrm{~S})$ cross section ratios in protonproton collisions at $\sqrt{s}=13 \mathrm{TeV}$, Phys. Rev. D 102 (2020) 092007

[6] CMS Collaboration, Observation of a New Excited Beauty Strange Baryon Decaying to $\Xi_{\mathrm{b}}^{-} \pi^{+} \pi$, Phys. Rev. Lett. 126 (2021) 252003

[7] ATLAS Collaboration, Observation of an Excited $\mathrm{B}_{c}^{ \pm}$Meson State with the ATLAS Detector, Phys. Rev. Lett. 113 (2014) 212004

[8] LHCb Collaboration, Search for excited $\mathrm{B}_{\mathrm{c}}^{+}$states, JHEP 01 (2018) 138

[9] CMS Collaboration, Observation of Two Excited $\mathrm{B}_{\mathrm{c}}^{+}$States and Measurement of the $\mathrm{B}_{\mathrm{c}}^{+}(2 \mathrm{~S})$ Mass in pp Collisions at $\sqrt{s}=13 \mathrm{TeV}$, Phys. Rev. Lett. 122 (2019) 132001

[10] LHCb Collaboration, Observation of an Excited $\mathrm{B}_{\mathrm{c}}^{+}$State, Phys. Rev. Lett. 122 (2019) 232001

[11] Particle Data Group, M. Tanabashi et al., Review of particle physics, Phys. Rev. D 98 (2018) 030001

[12] CMS Collaboration, Observation of a New $\Xi_{b}$ Baryon, Phys. Rev. Lett. 108 (2012) 252002

[13] LHCb Collaboration, Observation of Two New $\Xi_{\mathrm{b}}^{-}$Baryon Resonances, Phys. Rev. Lett. 114 (2015) 062004

[14] LHCb Collaboration, Observation of a New $\Xi_{\mathrm{b}}^{-}$Resonance, Phys. Rev. Lett. 121 (2018) 072002

[15] LHCb Collaboration, Observation of a new $\Xi_{b}^{0}$ state, Phys. Rev. D 103 (2021) 012004 